\title{
El significado semántico de la investigación científica en los institutos de educación superior tecnológica de Veracruz, México
}

\author{
The semantic meaning of scientific research at the institutes of higher \\ technological education in Veracruz, Mexico
}

ISSN 1510-2432 - ISSN 1688-9304 (en línea) - DOI: https://doi.org/10.18861/cied.2018.9.1.2820

Manuel Villarruel Fuentes

Doctor en Educación, Profesor e Investigador, Instituto Tecnológico de Úrsulo Galván, México. Responsable, Programas de Investigación en educación, ciencia, sociedad y tecnología para un desarrollo humano sostenido, y aprovechamiento holístico de los recursos forestales forrajeros como respuesta ecológica y productiva. Investigador, Sistema Nacional de Investigadores de México. Miembro, Consejo Mexicano de Investigación Educativa.

Fecha de recibido: $21 / 11 / 2017$

Fecha de aceptado: 31/03/2018

\begin{abstract}
Resumen
Actualmente la ciencia, en su calidad de pensamiento dominante, ha impactado de manera decidida en los modelos y enfoques educativos en el nivel superior. Esto ha condicionado el desarrollo de propuestas curriculares y didácticas, que en mayor o menor medida han sido puestas en marcha en los distintos niveles educativos de México, sin que a la fecha exista evidencia del logro efectivo de sus propósitos y metas. Tal es el caso del nivel superior tecnológico (Tecnológico Nacional de México) que desde 2012 ha desplegado un modelo educativo integral en todas sus instituciones. Bajo este contexto se hace necesario un acercamiento que, desde distintos ángulos, explore el fenómeno educativo asociado a la formación científica del estudiante así como la valoración que este hace de su papel en dicho proceso y de la investigación que se realiza dentro de sus planteles. Con base en ello se realizó un estudio bajo la técnica del Diferencial Semántico con el fin de identificar las percepciones que los estudiantes tienen sobre estos aspectos de su alfabetización científica. El estudio consideró un total de 835 estudiantes registrados (regulares) provenientes de 9 planteles (33\% del total), inscritos dentro del estado de Veracruz, México. Los resultados mostraron que los estudiantes tienen una imagen poco positiva de sí mismos y de sus compañeros, así como de la educación científica que están recibiendo al igual que sobre la investigación que se realiza dentro de los planteles.

Palabras clave: ciencia, investigación, educación superior, educación tecnológica.
\end{abstract}

\begin{abstract}
Science today, as dominant thinking, has impacted decisively on models and educational approaches at higher level. This has conditioned the development of curricular and didactic proposals, which to a greater or lesser extent have been implemented in the different levels of education in Mexico, in spite of the lack, to this date, of significant evidence of an effective achievement of its purposes and goals. Such is the case of the higher level in technology (National Institute of Technology of Mexico), which since 2012 has deployed an integral educational model in all its institutions. In this context, it is necessary an approach from different angles that explores both the educational phenomenon associated with the scientific training of the student and the assessment that students make of their role
\end{abstract}


in this process and of the research that is carried out within their schools. Based on this concept, a study has been conducted under the Semantic Differential technique, aiming at identifying the perceptions of students about such aspects of their scientific literacy. The study covered a total of 835 registered students (regular), from 9 schools (33\% of total), registered in the State of Veracruz, Mexico. The results showed that students have a low image of themselves and their classmates, as well as of the scientific education they are receiving and the research carried out within the campuses.

Keywords: Science, Research, Higher Education, Technological Education.

\section{Introducción}

Durante la segunda mitad del siglo XX los esquemas de organización social y los sistemas políticos dominantes construyeron el ideal de un mundo global en pleno desarrollo donde los indicadores de progreso y bienestar fueron concebidos a partir del paradigma científicotecnológico, quien se alimentó de las premisas de un saber verdadero auspiciado por el imperio de la razón. Prácticamente no existieron cuestionamientos acerca de las bondades de este modelo, cuyo ascetismo metodológico avaló toda invención humana, incluso aquellas encaminadas a la explotación de los recursos y la alteración de las condiciones de vida en el planeta.

Más allá de estos escenarios, de sus antecedentes recuperados desde la modernidad y de los entornos en que se concretó esta cosmovisión, lo cierto es que la ciencia, es su acepción tradicional, ha sido la vía para acceder a formas elaboradas de pensamiento, sirviendo de sustrato para desarrollar nuevas teorías que explican la conducta humana, los procesos cognitivos y metacognitivos, los fenómenos naturales, sociales y culturales, así como una amplia gama de cuestiones que han llevado al ser humano a sostener las actuales tesis y antítesis que hoy dominan el escenario ideológico-conceptual. Solo la ciencia es capaz de generar su propia némesis.

Ante este contexto, las instituciones de educación superior (IES) se han visto obligadas a sostener sus propuestas formativas sobre la base de los postulados y axiomas científicos, quienes le dictan, directa o indirectamente, cómo se debe enseñar, quién debe hacerlo, qué contenidos son los más adecuados, qué propósitos debe tener la educación, cuáles son las características que debe reunir un ser humano para ser integral, y lo que es más: qué sociedad es a la que se debe aspirar. Aquí es donde la ciencia toma dimensiones extraordinarias al fomentar la idea de un currículo por disciplinas, segmentador del conocimiento, que facilita el análisis de los campos cognitivos a partir de su departamentalización. Vessuri (2003) lo esclarece al señalar que:

“La dinámica cognitiva de la ciencia y su organización social convergieron en la creación de la ciencia pura, básica, fundamental, académica, a través de la constitución de disciplinas científicas y de modalidades de producción, difusión y legitimación del conocimiento científico que se volvieron estándar". (p.2)

Si como sugiere Vessuri (2003), las tecnologías se han constituido en el núcleo central de las políticas de desarrollo, entonces es fácil advertir la manera en que su asociación con la ciencia ha derivado en un paradigma científico-tecnológico que explica hoy el saber institucionalizado y corporativo que predomina en las IES sin que a la fecha existan estudios que permitan identificar la percepción que tiene el estudiante de nivel superior acerca de la investigación que se realiza en los centros escolares, la educación científica que recibe y el papel que a su juicio desempeña en dicho proceso. 
Por estas razones es necesario un acercamiento ordenado con los postulados que hacen posible entender la educación superior, sobre todo en el sistema de educación tecnológica -hoy Tecnológico Nacional de México-, donde predominan las propuestas curriculares basadas en una educación que se declara científica. Al respecto, el Modelo Educativo del Siglo XXI desarrollado por el Tecnológico Nacional de México (2012) puntualiza lo siguiente:

“El estudio de la tecnología debe desarrollar en los estudiantes una visión reflexiva y crítica, de cara a los vínculos entre la tecnología como producto cultural y la sociedad que se favorece o afecta por el uso o creación tecnológica. La actividad en ciencia y tecnología es un proceso social que produce efectos para el medio, por ello es importante que los individuos cooperen en la evaluación y el control de la actividad tecnológica; y que los jóvenes se inicien en la comprensión, participación y deliberación sobre temas relacionados con la tecnología". (p.61)

La pregunta es si los jóvenes estudiantes en realidad se interesan por los temas relacionados con la ciencia y la tecnología, dato que se evidencia a partir de su valoración de la educación científica que reciben dentro de los planteles y de la investigación que ahí se desarrolla, sin descartar la autovaloración que hacen de su papel en dichos procesos.

\section{Diseño metodológico}

La investigación se realizó como parte del proyecto denominado "Percepciones sociales en torno a la ciencia y la tecnología en instituciones de Educación Superior Tecnológica de Veracruz", financiado por el Tecnológico Nacional de México dentro de la línea "Educación, ciencia, sociedad y tecnología para un desarrollo humano sostenible" que se desarrolla en el Instituto Tecnológico de Úrsulo Galván.

\section{Población objeto de estudio y muestra}

La población estuvo integrada por el total de los estudiantes que cursaban el último año de una carrera profesional dentro de los institutos tecnológicos localizados en el estado de Veracruz, México, (estimado en 9 285), tanto federales como estatales. La muestra obtenida bajo muestreo no probabilístico, definido como de conveniencia (Casal y Mateu, 2003), constó de 835 estudiantes registrados (regulares) provenientes de 9 planteles (33\% del total), quienes habían cursado al menos uno de los dos talleres de investigación incluidos dentro de los programas académicos de todas las carreras (marco muestral). De acuerdo con Martínez-Salgado (2012) “...este tipo de procedimiento es que el tamaño de la muestra no se conoce al inicio, sino solo cuando la indagación ha culminado" (p. 617). Con este tamaño de muestra se obtuvo la saturación requerida para el estudio.

\section{Instrumento de evaluación}

El instrumento de evaluación se basó en la técnica del Diferencial Semántico, que en conceptos de Osgood citado por Pérez-Ortega et al., (2008):

“...se centra en el concepto o la percepción que tiene un individuo, cuando se le pide que valore un concepto en términos de un conjunto de dimensiones, tales como justoinjusto (evaluación de acción), fuerte-débil (percepción de su fuerza-potencia) y sensibleinsensible (percepción de su nivel de afecto-motivación)". (p.17) 
De acuerdo con Casas García, Alzás García y Casas García (2015), “El diferencial semántico (DS en adelante) mide las reacciones de las personas a palabras y conceptos de estímulo, en términos de estimaciones sobre escalas bipolares definidas por adjetivos antónimos". (p.37).

A decir de Arce-Fernández et al. (1997, p. 541) “El Diferencial Semántico (Osgood, Suci y Tannenbaum, 1957) quizá sea la técnica que más se ha utilizado en la investigación social para la medición de las actitudes. Se ha demostrado su eficacia repetidamente".

Lancheros et al. (2007) señalan que una actitud es una predisposición a responder positiva o negativamente a ciertos objetos o situaciones. Cuando se habla de objeto los autores precisan que se trata de personas, grupos, instituciones, organizaciones y problemas factibles de debate mientras que las situaciones se refieren a sucesos o comportamientos específicos de los objetos. Según Hogan (citado por Lancheros et al., 2007), existe consenso en que las actitudes tienen tres componentes: (a) cognitivo, que se compone de ideas articuladas y conscientes sobre el objeto; (b) emocional, referente a las sensaciones respecto al objeto; y (c) conductual, que consiste en las acciones emprendidas o con probabilidades de emprenderse con relación al objeto.

Sobre esta misma vertiente Pozo (1996) puntualiza que las actitudes tienen un componente conductual (formas de comportamiento), un componente afectivo (preferencias y rechazos) y un componente cognitivo (conocimientos y creencias). Sobre esta base las actitudes de un sujeto son más consistentes y estables cuando lo que realiza está de acuerdo con sus gustos y creencias.

Con todo ello Lancheros et al. (2007) precisan que no se debe confundir el concepto de actitud con el de interés, opinión, creencia o valor, ya que estos términos son utilizados de forma diferente, tal como lo demuestra la afirmación de Vicente, Cornejo y Barbero (2006), quienes puntualizan que:

“EI DS mide las reacciones valorativas de los sujetos a una gran variedad de estímulos verbales, conceptuales, objetos, identidades, personas, roles sociales, conductas, situaciones, colectivos, grupos, etc. en términos de puntuaciones en escalas bipolares definidas por contraste entre adjetivos contrapuestos relativos a los distintos factores valorados". (p.303)

Por su parte Malavé y Piñango (2012) determinan que:

“EI DS mide reacciones de las personas a estímulos (palabras, conceptos, imágenes) en forma de puntajes registrados en escalas bipolares, usualmente de siete intervalos con un punto neutral. Así, las escalas permiten medir la dirección y la intensidad de las reacciones. Los puntajes pueden combinarse de diversas maneras para describir y analizar los sentimientos de las personas..." (p.683)

Sin embargo, al final dichos autores asocian el diferencial semántico con medición de las actitudes hacia diversos objetos sociales.

Más allá de esta aparente confusión es relevante considerar que existe evidencia de que las actitudes pueden ser determinantes dentro de los procesos de aprendizaje, y como estas pueden ser aprendidas y modificadas, se constituyen en objetivos factibles de lograr (Vázquez y Manassero-Más, 1997) 
Basado en el potencial de la prueba, para este estudio el diferencial semántico se construyó con base en la estructura tridimensional EPA (evaluación, potencia y actividad) a fin de medir el significado connotativo de diversos estímulos o conceptos. Una gran cantidad de estudios demuestran que la estructura EPA es válida para estudiar tanto sujetos como conceptos y escalas, medidos a partir de sus pesos factoriales o espacios semánticos (distancias semánticas) entendidas como la distancia que existe entre el concepto y el punto neutral del espacio tridimensional. Con base en ello es posible medir la afectación total de un estímulo y comparar su semejanza con otros estímulos.

Para efectos de la interpretación de la estructura, Charia (2015) explica el papel de cada dimensión incluida que desglosa de la siguiente manera:

- Evaluación. Realizada por el individuo a partir del concepto que está analizando o clasificando.

- Potencia. Entendida como el poder del concepto que percibe el individuo.

- Actividad. Percibida por el individuo acerca del objeto o concepto.

La estrategia de aplicación consistió en presentar por separado los conceptos y sus escalas, pero seguidas una de otra, alternando los polos de su dirección. En total se presentaron tres conceptos con 12 escalas cada uno. Dichos conceptos fueron: 1) La educación científica que se recibe dentro del tecnológico es...; 2) El estudiante del instituto tecnológico es...; 3) La investigación que se realiza dentro del tecnológico es... Los dos primeros conceptos bajo escala positivo-negativo y el tercero negativo-positivo. Las escalas abarcaron siete niveles o intervalos (1 al 7) (Tabla 1). La confiabilidad consideró la prueba Alfa de Cronbach (0.70). En busca de estimar la varianza de los factores se estimó la validez de las escalas semánticas mediante el análisis de componentes principales (López-González y AnayaRodríguez, 2016). 
Tabla 1. Conceptos presentados como diferenciales semánticos a estudiantes del nivel superior tecnológico de Veracruz, México.

\begin{tabular}{|c|c|c|c|c|c|c|c|c|}
\hline \multicolumn{9}{|c|}{ 1. La educación científica que se recibe dentro del tecnológico es: } \\
\hline & 1 & 2 & 3 & 4 & 5 & 6 & 7 & \\
\hline Intensa & & & & & & & & Débil \\
\hline Profunda & & & & & & & & Superficial \\
\hline Pesada & & & & & & & & Ligera \\
\hline Rápida & & & & & & & & Lenta \\
\hline Ardua & & & & & & & & Fácil \\
\hline Organizada & & & & & & & & Desorganizada \\
\hline Urgente & & & & & & & & Parsimoniosa \\
\hline Activa & & & & & & & & Pasiva \\
\hline Compleja & & & & & & & & Simple \\
\hline Entretenida & & & & & & & & Aburrida \\
\hline Valiosa & & & & & & & & Sin valor \\
\hline Formal & & & & & & & & Informal \\
\hline \multicolumn{9}{|c|}{ 2. El estudiante del instituto tecnológico es: } \\
\hline & 1 & 2 & 3 & 4 & 5 & 6 & 7 & \\
\hline Profundo & & & & & & & & Superficial \\
\hline Intenso & & & & & & & & Débil \\
\hline Pesado & & & & & & & & Ligero \\
\hline Resistente & & & & & & & & Frágil \\
\hline Tenaz & & & & & & & & Displicente \\
\hline Organizado & & & & & & & & Desordenado \\
\hline Atento & & & & & & & & Disipado \\
\hline Activo & & & & & & & & Pasivo \\
\hline Innovador & & & & & & & & Tradicional \\
\hline Fiable & & & & & & & & Inseguro \\
\hline Rebelde & & & & & & & & Obediente \\
\hline Eficiente & & & & & & & & Ineficiente \\
\hline \multicolumn{9}{|c|}{ 3. La investigación que se realiza dentro del tecnológico es: } \\
\hline & 1 & 2 & 3 & 4 & 5 & 6 & 7 & \\
\hline Inestable & & & & & & & & Estable \\
\hline Lenta & & & & & & & & Rápida \\
\hline Floja & & & & & & & & Compacta \\
\hline Débil & & & & & & & & Fuerte \\
\hline Sencilla & & & & & & & & Compleja \\
\hline Conservadora & & & & & & & & Renovadora \\
\hline Rígida & & & & & & & & Manipulable \\
\hline Desordenada & & & & & & & & Ordenada \\
\hline Fea & & & & & & & & Bella \\
\hline Inaccesible & & & & & & & & Accesible \\
\hline Seria & & & & & & & & Alegre \\
\hline Peligrosa & & & & & & & & Segura \\
\hline
\end{tabular}




\section{Análisis estadístico}

Para el análisis estadístico se empleó la hoja de cálculo del programa Excel, del software office de Windows versión 10. Se determinó la Puntuación Factorial por cada dimensión (EPA) así como el Índice de Polarización por cada concepto o cuestionamiento planteado. Con fines de comparación se estimaron la distancia semántica y los promedios para cada concepto.

\section{Resultados y discusión}

En la Figura 1 se muestra la puntuación media para las tres dimensiones EPA (evaluación, potencia y actividad) para cada uno de los conceptos planteados a los estudiantes. Bajo este perfil es posible destacar el valor que los estudiantes otorgan tanto a la educación científica que se recibe dentro de los institutos tecnológicos como a su papel dentro de las dinámicas académicas desarrolladas por ellos dentro de los tecnológicos del estado de Veracruz. Al considerar que la escala fue del 1 al 7 siendo 7 lo deseable, pone en relieve un precario concepto sobre la importancia de su papel (=4.975) que, sumado a lo mostrado sobre su educación científica $(=4.536)$, lleva a recomendar un mayor y mejor involucramiento de los estudiantes en tareas didácticas proclives a su alfabetización científica y al desarrollo de mejores proyectos de investigación que en calidad de escenarios para el aprendizaje propicien en el estudiante un mayor interés y valoración de su proceso formativo.

Aunado a ello, la valoración que hacen de la investigación que allí se realiza (siendo 7 lo deseable al invertirse el sentido de la escala de negativa a positiva), se muestra también deficiente $(=3.777$, cercano al punto central de la escala), lo que plantea la necesidad de establecer un programa para el fomento de la investigación científica a través de proyectos que integren con mayor dinamismo a los estudiantes del sistema tecnológico.

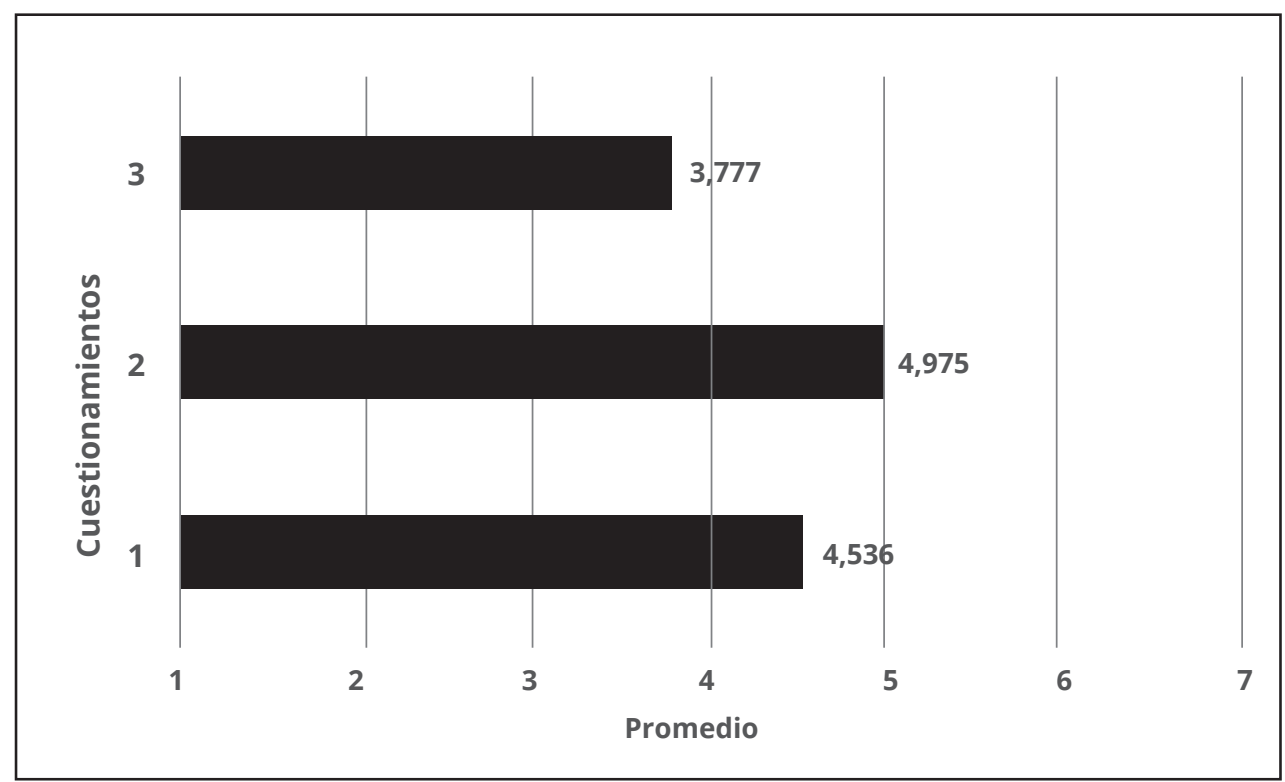

Figura 1. Promedios generales encontrados en torno a los cuestionamientos hechos a los estudiantes del nivel superior tecnológico de Veracruz, México. 
Al revisar las cargas factoriales hacia el interior de cada concepto se aprecian puntuaciones muy cercanas entre sí, tanto para la potencia como para la acción y la evaluación (Tabla 2), lo que permite pensar en la existencia de una equilibrada actitud hacia las actividades que realizan los estudiantes del nivel superior tecnológico de Veracruz, así como para la mostrada hacia la educación científica. Al respecto Romero-Bojórquez, Utrilla-Quiroz y Utrilla-Quiroz, 2014) conciben las actitudes como:

“...la evaluación que hacen los sujetos de los eventos de la vida cotidiana que pueden ser positivos o negativos, dándoles un sesgo emocional al aprendizaje. Lo cognitivo y lo emocional participan en la manifestación de conductas (actitudes), que favorecen u obstaculizan el aprendizaje en el aula escolar". (p.292)

Aunado a ello se mostró una equilibrada puntuación factorial para la investigación que se efectúa dentro de los tecnológicos (3.965, 3.799 y 3.567 para potencia, acción y evaluación respectivamente). Esto pone de manifiesto que la estructura del significado es prácticamente igual para las tres dimensiones del concepto propuesto en este estudio.

Aunque el mayor Índice de Polarización (Tabla 2) fue para el cuestionamiento relativo al papel del estudiante (8.621), seguido del relativo a la educación científica (7.858) y la investigación que se realiza (6.549), las distancias semánticas de este diferencial con respecto al cuestionamiento sobre la educación científica y el papel del estudiante se mostraron cercanas (0.587) (Tabla 3), no así para el cuestionamiento sobre el rol del estudiante y la investigación científica cuya distancia fue de 2.119. Este hallazgo deja entrever ciertas discrepancias entre estos dos cuestionamientos (estímulos), vistos como distantes por los estudiantes del nivel superior tecnológico en Veracruz. Finalmente, la distancia semántica entre la educación científica y la investigación se mostró relevante (1.367), lo que permite vislumbrar una percepción que separa estos conceptos, como si se tratara de dos entidades distintas y no asociadas.

Tabla 2. Cargas factoriales e índices de polarización de los cuestionamientos hechos a los estudiantes del nivel superior tecnológico de Veracruz.

\begin{tabular}{|l|l|l|l|l|}
\hline & $\begin{array}{l}\text { Cuestionamientos } \\
\text { (conceptos) }\end{array}$ & Dimensiones & $\begin{array}{l}\text { Puntuación } \\
\text { factorial }\end{array}$ & $\begin{array}{l}\text { Índice de } \\
\text { polarización }\end{array}$ \\
\hline $\begin{array}{l}\text { (+/1) } \\
(-/ 7)\end{array}$ & $\begin{array}{l}\text { La educación científica que se } \\
\text { recibe dentro del tecnológico es: }\end{array}$ & $\begin{array}{l}\text { Acción } \\
\text { Evaluación }\end{array}$ & 4.421 & 7.858 \\
\hline $\begin{array}{l}(-/ 1) \\
\text { (+/7) }\end{array}$ & La investigación que se realiza & Potencia & 4.672 & 3.965 \\
\hline $\begin{array}{l}\text { dentro del tecnológico es: } \\
(+/ 1)\end{array}$ & Ección & 3.799 & 6.549 \\
$(-/ 7)$ & El estudiante del instituto & Potencia & 3.567 & \\
\hline & tecnológico es: & Acción & 4.811 & 8.621 \\
\hline
\end{tabular}


Tabla 3. Distancias semánticas entre los cuestionamientos hechos a los estudiantes del nivel superior tecnológico de Veracruz.

\begin{tabular}{|l|l|}
\hline Cuestionamiento & Distancia semántica (unidades) \\
\hline 1 vs 2 & 0.587 \\
1 vs 3 & 1.367 \\
2 vs 3 & 2.119 \\
\hline
\end{tabular}

1. La educación científica que se recibe dentro del tecnológico; 2. El estudiante del instituto tecnológico; 3. La investigación que se realiza dentro del tecnológico.

Con todo ello, las distancias semánticas no evidencian discrepancias importantes en el sentido connotativo que se le otorga al primer y segundo cuestionamiento (educación científica y estudiante), por lo que es posible afirmar que la percepción que tienen los estudiantes es muy cercana para estas dos interrogantes; no así para la educación científica y la investigación, y entre esta última y el estudiante.

La relevancia de estos hallazgos se hace evidente al revisar lo establecido por RojasBetancur, Méndez-Villamizar y Rodríguez-Prada (2012), quienes señalan lo siguiente:

“En la investigación educativa, el estudio de la actitud hacia la ciencia en general y hacia la investigación científica en particular, está íntimamente relacionado con la existencia de una formación crítica, revitalizada en el desarrollo de las capacidades de profesores y de estudiantes de elaborar el conocimiento de una manera dinámica, provocadora, y que impulse la pregunta científica como centro de la formación profesional". (p.217)

Como lo expresan estos autores, “...la mejor actitud hacia la investigación será un dispositivo necesario en el estudiante para lograr una mejor formación científica y una mayor posibilidad de ampliación de las comunidades académicas del país..." (p.217), condición que al parecer puede estar en cierta medida comprometida dentro del sistema de educación tecnológica.

Sobre esta base es indispensable asumir una nueva forma de orientar la educación científica y el quehacer de la investigación que se desarrolla en el sistema educativo tecnológico en Veracruz, recuperando los preceptos de Rojas-Betancur et al. (2012) anteriormente citados. Se trata de un abordaje que no desestima el pensamiento algorítmico pero que impulsa y privilegia los abordajes heurísticos en busca de alcanzar el logro de sólidos hábitos de estudio, entendidos como los ejes coyunturales que definen la actitud a desplegar.

Vélez-Ramírez (2008) define con amplitud esta condición, al señalar que:

“Frente a la crisis que vive la educación superior, estimo que los hábitos de estudio pueden constituirse en una estrategia que afecte la relación que los alumnos y profesores establecen con el conocimiento, es decir, con los hábitos intelectuales, que -conviene insistir- solo se adquieren al alcanzar la verdad. También como una manera de dinamizar la institución [...], ya que permiten nuevas formas de enseñanza en los tradicionales espacios académicos, cualificando la docencia al favorecer la investigación y al hacer que esta sea labor cotidiana de profesores y estudiantes". (p.169) 
Pero no pueden desarrollarse dichos hábitos de estudio distanciados de los hábitos intelectuales asociados al pensamiento científico y a la investigación, como tampoco pueden darse estos sin una cultura científica que los posibilite y fomente. Una cultura que, a decir de Vázquez \& Manassero-Más (1995), se sustente en una orientación crítica y humanística desprovista de distinciones o discriminaciones. Vinculado a ello, Hilarraza (2012) propone el diseño y despliegue de una pedagogía de la investigación que, a manera de estrategia, permita la enseñanza del método científico integrándose un proceso virtuoso que, en conceptos de Olmedo (2011), impulse la indagación y la transformación del conocimiento. Sin duda esto no es fácil de alcanzar, ya que dentro de los institutos tecnológicos de nivel superior en México se asume como implícito el desarrollo de un pensamiento científico y las habilidades para la investigación con solo agregar dos o tres asignaturas dentro de los programas académicos que se desarrollan, comunes a todos los campos disciplinarios, todavía lejos de una pedagogía y una didáctica para la enseñanza-aprendizaje de la ciencia que les acompañe. Esto sin duda debe ser atendido en el corto plazo. Los resultados encontrados en este estudio confirman la urgencia de su cumplimiento.

\section{Conclusiones}

Es posible afirmar que la estructura conceptual tridimensional de los estudiantes con respecto a los tres estímulos planteados se mostró compacta hacia el interior de cada una de ellas (potencia, acción y evaluación). En este sentido las discrepancias entre las percepciones muestran una alineación conceptual no aceptable.

Los estudiantes mostraron una imagen disminuida de sí mismos y de sus compañeros dentro de la actividad científica que desarrollan así como de la educación científica que reciben, al igual que de la investigación que se realiza dentro de los planteles, situación que debe estar afectando la actitud con la cual enfrentan los estudiantes su proceso formativo y su desempeño dentro del campo de la investigación científica. Sobre el particular, Romero-Bojórquez, Utrilla-Quiroz y Utrilla-Quiroz (2014) dejan en claro que, “En el contexto escolar somos los profesores y alumnos quienes construimos el sentido y el significado de los procesos de enseñanza y aprendizaje con nuestras interacciones en el aula" (p.293), por lo que se debe prestar mayor atención a la interacción docenteestudiante, particularmente en el terreno de la alfabetización científica y el desarrollo de proyectos en conjunto, que en la educación superior tecnológica suele basarse en el trabajo de campo, taller y laboratorio. Los mismos autores reportan que dentro de los procesos de enseñanza-aprendizaje que tienen lugar en ambientes escolarizados existe evidencia de que los profesores manifiestan actitudes que incrementan la inseguridad de los estudiantes, generando climas emocionalmente negativos. Como ejemplos citan los discursos docentes donde se anticipa a los estudiantes la complejidad de la materia, lo difícil de sus contenidos y lo inminente de su fracaso escolar.

Si, como lo señalan García-Sánchez, Aguilera-Terrats y Castillo-Rosas (2011), "las actitudes están relacionadas con el comportamiento que mantenemos en torno a los objetos a que hacen referencia" (p.2), entonces existe un amplio margen de mejora en estos tres rubros, sobre todo en el reforzamiento de las percepciones (y con ello las actitudes) sobre la investigación que tiene lugar habitualmente en el nivel superior tecnológico de Veracruz. Si la actitud de los estudiantes hacia la investigación es desfavorable, entonces es probable que no se inclinen hacia esta actividad, ya que, si bien las actitudes no reflejan fielmente la conducta, si son un indicador importante de ella. 
Estos resultados confirman lo encontrado en la primera fase del proyecto sobre percepciones sociales, del que formó parte este estudio, donde se evidenciaron precarias percepciones sociales acerca de la ciencia y la tecnología, en las distintas categorías evaluadas (saberes, valores y creencias) (Villarruel-Fuentes et al., 2017).

\section{Referencias bibliográficas}

Arce-Fernández, C., Stem, D. E., Andrade-Fernández, E. M. y Seoane-Pesqueira, G. (1997). Comparación de escalas de respuesta para la medición de las actitudes. Psicothema, 9 (3), 541-545. Recuperado de: http://www.redalyc.org/pdf/727/72709308.pdf

Casal, J. y Mateu, E. (2003). Tipos de muestreo. Rev. Epidem. Med. Prev. 1, 3-7.

Casas García, J. A., Alzás García, T. y Casas García, L. M. (2015). Actitudes hacia la sexualidad en estudiantes de enfermería: Un análisis con Diferencial Semántico y Redes Asociativas Pathfinder. Revista de Psicología y Educación, 10(1), 33-54. Recuperado de: http://www. revistadepsicologiayeducacion.es/pdf/116.pdf

Charia, H. (2015). Estudio comparado de la percepción del estado de salud, del dolor crónico y de la imagen corporal en mujeres universitarias marroquíes y españolas. Tesis Doctoral, Universidad de Alicante. 239 p. Recuperado de: https://rua.ua.es/dspace/ bitstream/10045/54567/1/tesis_hikmat_charia.pdf

García-Sánchez, J., Aguilera-Terrats, J. R. y Castillo-Rosas, A. (2011). Guía técnica para la construcción de escalas de actitud. Odiseo, Revista Electrónica de Pedagogía, 8 (16), 1-13. Recuperado de: http://www.odiseo.com.mx/2011/8-16/garcia-aguilera-castillo-guiaconstruccion-escalas-actitud.html

Hilarraza, Y. J. (2012). La investigación pedagógica: un aporte a la gestión de la formación docente desde un punto de vista socio cultural.Revista Didasc@lia: didáctica y educación, 3 (1), 25-39.

Lancheros, L., Marconi, L., Manrique, M. \& Mendivelso, M. (2007). Conceptos básicos acerca de las pruebas de actitud. Avances en medición, 5, 163-167.

López-González, R. y Anaya-Rodríguez, R. (2016). Estudiantes universitarios interactuando en red: ¿nuevos escenarios de interacción, expresión y participación ciudadana? Revista interamericana de educación de adultos, 38 (1), 49-67. Recuperado de: http://www.crefal. edu.mx/rieda/images/rieda-2016-1/exploraciones2.pdf

Malavé, J. y Piñango, R. (2012). Líderes, gerentes y emprendedores: un estudio empírico de actitudes y significados. Revista Venezolana de Gerencia, 17 (60), 676-694. Recuperado de: http://www.redalyc.org/pdf/290/29024892007.pdf

Martínez-Salgado, C. (2012). El muestreo en investigación cualitativa. Principios básicos y algunas controversias. Ciência \& Saúde Coletiva, 17 (3), 613-619. Disponible en: http://www. scielo.br/pdf/csc/v17n3/v17n3a06.pdf

Olmedo-Estrada, J. C. (2011). Educación y divulgación de la ciencia: tendiendo puentes hacia la alfabetización científica. Revista Eureka sobre enseñanza y divulgación de las ciencias, 8 (2), 137-148. 
Pérez-Ortega, G., Arango-Serna, M. D. y Branch-Bedoya, J. W. (2008). El semántico diferencial como propuesta metodológica para caracterizar el liderazgo en una organización. Dyna, 75 (155), 15-27. Disponible en: http://www.redalyc.org/pdf/496/49611953003.pdf

Pozo, J. I. (1996). Aprendices y maestros, Madrid: Alianza.

Rojas-Betancur, H. M., Méndez-Villamizar, R. y Rodríguez-Prada, Á. (2012). Índice de actitud hacia la investigación en estudiantes del nivel de pregrado. Entramado, 8 (2), 216-229. Recuperado de: http://www.redalyc.org/pdf/2654/265425848014.pdf

Romero-Bojórquez, L., Utrilla-Quiroz, A. y Utrilla-Quiroz, V. M. (2014). Las actitudes positivas y negativas de los estudiantes en el aprendizaje de las matemáticas, su impacto en la reprobación y la eficiencia terminal. RaXimhai, 10 (5), 291-319. Recuperado de: http://www. redalyc.org/pdf/461/46132134020.pdf

Tecnológico Nacional de México (2012). Modelo educativo para el siglo XXI. Formación y desarrollo de competencias profesionales. México: Dirección General de Educación Superior Tecnológica. Recuperado de: http://www.itmexicali.edu.mx/informacion/modelo_educativo. pdf

Vázquez, Alonso, A. y Manassero-Más, M. A. (1997). Una evaluación de las actitudes relacionadas con la ciencia. Enseñanza de las Ciencias, 15 (2), 199-213.

Vázquez-Alonso, A., \& Manassero-Mas, M. A. (1995). Actitudes relacionadas con la ciencia: una revisión conceptual. Enseñanza de las Ciencias, 13 (3), 337-346. Disponible en: https:// ddd.uab.cat/pub/edlc/02124521v13n3/02124521v13n3p337.pdf

Vélez-Ramírez, A. (2008). La adquisición de hábitos como finalidad de la educación superior. Educación y educadores, 11 (1), 167-180. Recuperado de: http://educacionyeducadores. unisabana.edu.co/index.php/eye/article/viewFile/723/806

Vessuri, H. (2003). La ciencia y la educación superior en el proceso de internacionalización. Elementos de un marco conceptual para América Latina. Unesco, Forum Occasional Paper Series. Paper no. 3/S. París: Unesco. Recuperado de: http://unesdoc.unesco.org/ images/0013/001347/134783so.pdf

Vicente, R., Cornejo, J. M. y Barbero, F. (2006). La evaluación de la actividad grupal. El análisis de la actividad grupal (AAG) y la aplicación DSCLIMA del Sistema SOCIOS. Anuario de Psicología, 37 (3), 299-320. Recuperado de: http://www.raco.cat/index.php/ anuariopsicologia/article/viewFile/61843/82855

Villarruel-Fuentes, M., Pérez-Santiago, F., Chávez-Morales, R. y Hernández-Arano, I. (2017). Percepciones sobre ciencia y tecnología en estudiantes del nivel superior tecnológico de Veracruz, México. Revista perspectiva educacional. Formación de profesores, 56 (1), 43-61. Recuperado de: http://www.perspectivaeducacional.cl/index.php/peducacional/article/ viewFile/465/233 